\title{
CHANGES IN EMBRYONIC STEM CELL COLONY MORPHOLOGY AND EARLY DIFFERENTIATION MARKERS DRIVEN BY COLLOIDAL CRYSTAL TOPOGRAPHICAL CUES
}

\author{
Lijun Ji ${ }^{\S}$, Vanessa L.S. LaPointe ${ }^{\S}$, Nicholas D. Evans ${ }^{\#}$ and Molly M. Stevens*
}

\begin{abstract}
Departments of Materials and Bioengineering and the Institute of Biomedical Engineering, Imperial College London, London, SW7 2AZ, UK
\end{abstract}

\#Present address: Bone and Joint Research Group, University of Southampton School of Medicine, Southampton, SO16 6YD, UK

${ }^{\S} \mathrm{L}$. Ji and V. LaPointe contributed equally to this work.

\begin{abstract}
The use of materials properties to guide cell behaviour is an attractive option for regenerative medicine, where controlling stem cell behaviour is important for the establishment of a functioning cell population. A wide range of materials properties have been shown to influence many types of cells but little is known about the effects of topography on embryonic stem cells (ESCs). In order to advance this knowledge, we synthesised and characterised substrates formed of silica colloidal crystal (SCC) microspheres to present highly ordered and reproducible topographical features from $120-600 \mathrm{~nm}$ in diameter. We found that, compared to cells cultured on flat glass, cells cultured on the SCC substrates retained transcription of stem cell (Dppa5a, Nanog, and Pou5f1) and endoderm (Afp, Gata4, Sox17, and Foxa2) markers more similar to undifferentiated ESCs, suggesting the substrates are restricting differentiation, particularly towards the endoderm lineage. Additionally, five days after seeding, we observed strikingly different colony morphology, with cells on the SCC substrates growing in spherical colonies approximately ten cells thick, while cells on glass were growing in flat monolayers. Colonies on the SCC substrates developed a central pit, which was never observed in cells cultured on glass, and expressed proteins related to epithelialisation. Together, these data demonstrate the potential of using topographical cues to control stem cell behaviour in vitro.
\end{abstract}

Keywords: Embryonic stem cells, stem cell differentiation, silica colloidal microspheres, epithelialisation, tissue engineering.

\footnotetext{
*Address for correspondence:

Molly M. Stevens

Departments of Materials and Bioengineering

Prince Consort Road

Imperial College London,

London, SW7 2AZ, U.K.
}

E-mail: m.stevens@imperial.ac.uk

\section{Introduction}

Cell-substrate interactions play an important role in regulating and determining cell fate both in vivo and in vitro (Stevens and George, 2005). The prospect of controlling cell behaviour using engineered substrates is particularly attractive for both fundamental research and therapeutic applications. Engineered substrates may permit the elucidation of various adhesion-dependent signalling pathways to further our understanding of the influence of material properties on cell behaviour. This knowledge can be used to inform material design decisions for therapeutic applications such as in regenerative medicine. For these reasons, in recent years there has been considerable interest in tailoring substrate properties in order to generate a desired cell response.

Several biological and physical substrate properties are known to affect cell behaviour in vitro. It has been repeatedly demonstrated in both tissue engineering and developmental biology that many cell types require specific protein constituents in their extracellular matrices (ECM). For example, pancreatic $\beta$-cells encapsulated in hydrogels containing certain laminin-derived peptides demonstrated improved survival and function compared to cells in hydrogels without targeted cell-matrix interactions (Weber et al., 2007). Similarly, the self-renewal of murine embryonic stem cells (ESCs) has been shown to be regulated through integrin signalling, again underlining the importance of cell-matrix interactions on cell behaviour (Hayashi et al., 2007; Lee et al., 2010). In addition to these biological signals, many physical properties have been shown to affect cell behaviour. Substrate chemistry affects protein adsorption to subsequently influence adhesion (Keselowsky et al., 2005) and differentiation (Curran et al., 2005; Benoit et al., 2008; Curran et al., 2011). Substrate stiffness, or matrix elasticity, is one of the most widely studied properties and can direct both adult and stem cell lineage choice and differentiation (Engler et al., 2006; Discher et al., 2009; Evans et al., 2009; Gilbert et al., 2010).

Another well studied property is the role of surface topography, which is of particular interest due to its relevance to cell behaviour in vivo, where cells experience a variety of different topographies, such as the architecture of the basement membrane and differing orientations of ECM proteins (Frantz et al., 2010). Several methods, such as deposition techniques, lithography, and rapid prototyping have been developed in the last decade to 
create tailored substrates designed to probe the effects of topography on cells. Interestingly, different cell types respond differently to these topographical influences, potentially due to their widely varying extracellular environments in vivo (Rozario and DeSimone, 2010).

In elegant early work, micropatterned surfaces coated with ECM proteins were used to show that adhesion site spacing geometry could determine cell shape and whether individual cells grow or die (Chen et al., 1997). Further work in the field established the relationship between cell shape and differentiation in mesenchymal stem cells (MSCs). MSC differentiation is now known to be governable by cell shape independent of soluble factors (McBeath et al., 2004; Kilian et al., 2010; Unadkat et al., 2011). Furthermore, topographical features on the nanometre scale have been shown to affect osteogenic differentiation (Dalby et al., 2007) and self-renewal of MSCs (McMurray et al., 2011). The combination of topographical features and chemical motifs has also been shown to direct MSC differentiation (Curran et al., 2010).

Embryonic stem cells (ESCs) are of particular interest as a potent cell source for regenerative medicine therapies due to their ability to differentiate into all somatic cell types found in the adult organism, and their sensitivity to small changes in culture conditions (Evans and Kaufman, 1981). In the presence of leukaemia inhibitory factor (LIF), a differentiation-inhibiting cytokine, murine ESCs can be maintained in culture in an undifferentiated state. Differentiation protocols in vitro typically involve the formation of embryoid bodies (EBs), which are aggregates of cells derived from ESCs that represent the earliest stages of differentiation. ESCs can also be differentiated on two-dimensional substrates in the presence of exogenous factors, such as soluble growth factors or substrateadsorbed ECM molecules (Salasznyk et al., 2004). Compared to other adherent cell types, little is known about the effects of substrate properties on ESC behaviour, perhaps because ESCs are typically grown on a layer of feeder cells, which effectively obscures the substrate.

We chose to study the effects of nano- to micrometre scale topography because the ECM and basement membrane are composed of a rich array of architectural features such as aligned fibres, interconnected pores, and ridges that range from 10-300 $\mathrm{nm}$ in size (Chai and Leong, 2007) and prior work has demonstrated the role of topography in guiding cell behaviour. Silica colloidal crystal (SCC) microsphere substrates were used to investigate the effects of topography on feeder-free ESCs. The substrates were conferred ordered hemispherical topography through the packing of microspheres of varying diameter and were highly reproducible and simple to fabricate. By examining colony morphology, gene expression, and protein expression, we herein test the hypothesis that these substrates can modulate ESC colony morphology and behaviour and hence provide a means to investigate the fundamental effects of topography on ESCs in vitro and to inform the rational design of biomaterials for regenerative medicine.

\section{Materials and Methods}

\section{Substrate fabrication}

Monodisperse silica microspheres (SCCs) with diameters of 120,400 , and $600 \mathrm{~nm}$ were synthesised through alkaline hydrolysis of tetraethoxysilane (Sigma-Aldrich, Dorset, UK; 99 \%) according to Stöber's method (Stöber et al., 1968). Briefly, aqueous ammonia was added to a mixture of ethanol and deionised water in a round bottom flask to obtain a $\mathrm{pH}$ of 9.0. The basic solution was stirred rigorously at ambient temperature, and tetraethoxysilane was added for hydrolysis. Silica microspheres formed in the solution in $3 \mathrm{~h}$. SCC substrates were then fabricated by drying the silica microsphere suspension, causing it to self-assemble into a closely packed substrate. In order to increase the mechanical competence of the substrates, the SCCs were sintered at $800{ }^{\circ} \mathrm{C}$ for $3 \mathrm{~h}$ to promote partial coalescence between the silica microspheres. The substrates comprised of approximately 120, 400 and $600 \mathrm{~nm}$ SCCs are referred to as SCC120, SCC400 and SCC600, respectively. SCC substrates and glass cover slips (used as a reference material for topography; Agar Scientific, Stansted, UK) with an area of approximately $1.3 \mathrm{~cm}^{2}$ were coated with collagen to permit cell adhesion by overnight immersion in an excess of $125 \mu \mathrm{g} / \mathrm{mL}$ collagen (rat tail collagen (Type I), First Link, Birmingham, UK) solution in HEPES buffer. Substrates were then dried at room temperature before cell seeding.

\section{Collagen quantification}

In order to confirm collagen adsorption was equal on both the SCC substrates and the glass cover slips, we quantified the absorption using a previously reported method based on the binding of biotinylated fibronectin to Type I collagen (Gaudet et al., 2003). Substrates were weighed and incubated for $30 \mathrm{~min}$ in a blocking buffer of $2 \%(\mathrm{w} / \mathrm{v})$ bovine serum albumin and $0.05 \%(\mathrm{v} / \mathrm{v})$ Tween-20 in PBS. They were then incubated for $1 \mathrm{~h}$ at $37{ }^{\circ} \mathrm{C}$ in a $0.2 \mu \mathrm{g} / \mathrm{mL}$ solution of fibronectin (SigmaAldrich) biotinylated with an EZ-Link ${ }^{\circledR}$ Sulfo-NHSBiotinylation Kit (Pierce Biotechnology/Thermo Scientific, Cramlington, UK) according to the manufacturer's instructions. Substrates were rinsed three times in 0.1 $\%(\mathrm{v} / \mathrm{v})$ Tween-20 in PBS and incubated in $50 \mathrm{ng} / \mathrm{mL}$ horseradish peroxidase-streptavidin in blocking buffer for $30 \mathrm{~min}$ at $37^{\circ} \mathrm{C}$. Substrates were rinsed three times and incubated in 3,3',5,5' -tetramethylbenzidine (TMB) for $10 \mathrm{~min}$ at room temperature. The reaction was stopped with an equal volume of $2 \mathrm{M} \mathrm{H}_{2} \mathrm{SO}_{4}$. After $30 \mathrm{~min}, 200$ $\mu \mathrm{L}$ of the mixture was transferred to a 96 -well plate and absorbance values were measured on a colorimetric plate reader at $450 \mathrm{~nm}$ (SpectraMax M5, Molecular Devices, Sunnyvale, CA, USA).

\section{Cell culture}

Cell culture reagents were from Invitrogen/Life Technologies (Bromborough, UK) unless specified otherwise. Murine embryonic stem cells (ESCs; ATCC CRL-1821 obtained from LGC Standards, Teddington, UK) were subcultured in growth medium (DMEM 
supplemented with $10 \%(\mathrm{v} / \mathrm{v})$ foetal bovine serum, $2 \mathrm{mM}$ L-glutamine, $50 \mathrm{U} / \mathrm{mL}$ penicillin, $50 \mathrm{mg} / \mathrm{mL}$ streptomycin, $100 \mu \mathrm{M} \beta$-mercaptoethanol (Sigma-Aldrich), and $1 \mu \mathrm{L} /$ $\mathrm{mL}$ conditioned medium from LIF-producing Cos-7 cells (homemade, plasmid kindly provided by Prof. Vasso Episkopou, Imperial College London, UK) at $37{ }^{\circ} \mathrm{C}$ and $5 \% \mathrm{CO}_{2}$. Cells were seeded on SCC substrates and cover slips at a density of approximately $2.7 \times 10^{5} \mathrm{cells} / \mathrm{cm}^{2}$ and cultured in growth medium for five days with daily medium changes, after which the cells were cultured in differentiation medium (growth medium without supplemented LIF or $\beta$-mercaptoethanol).

\section{Embryoid body formation}

Embryoid bodies (EBs) were formed by dispersing ESCs into aggregates of approximately $10-30$ cells by incubation with $0.05 \%(\mathrm{w} / \mathrm{v})$ trypsin-EDTA for $1 \mathrm{~min}$. Cell aggregates were pelleted by centrifugation at $50 \mathrm{x}$ g for $2 \mathrm{~min}$, and the aggregates were transferred to microbiological-grade Petri dishes (Bibby Sterilin/Thermo Fisher) and fed daily with the same medium as cells on the SCC substrates and glass cover slips.

\section{Quantitative real-time polymerase chain reaction (qPCR)}

At the indicated time points, RNA was isolated using an RNeasy mini kit (Qiagen, Hilden, Germany) according to the manufacturer's instructions. Sensimix OneStep kit (Quantace, Watford, UK) was used to perform both reverse transcription and qPCR. Thermocycling and SYBR Green detection were performed on a Corbett Rotorgene 6000 (Qiagen) with 40 cycles consisting of annealing at 57 ${ }^{\circ} \mathrm{C}$, extension at $72{ }^{\circ} \mathrm{C}$, and denaturing at $95^{\circ} \mathrm{C}$. Primers to Gapdh (forward: AACTTTGGCATTGTGGAAGG; reverse: ACACATTGGGGGTAGGAACA), Nanog (forward: TTGCTTACAAGGGTCTGCTACT; reverse: ACTGGTAGAAGAATCAGGGCT), Fgf5 (forward: TGTGTCTCAGGGGATTGTAGG; reverse: AGCTGTTTTCTTGGAATCTCTCC), and Foxa2 (forward: CCCTACGCCAACATGAACTCG; reverse: GTTCTGCCGGTAGAAAGGGA) were selected from Primer Bank (Harvard University, Cambridge, MA, USA; http://pga.mgh.harvard.edu/primerbank/). Transcript expression was calculated using the $\Delta \Delta \mathrm{C}_{\mathrm{t}}$ method relative to ESCs prior to seeding, with Gapdh as the internal control. Data are presented as $2^{-\Delta \Delta \mathrm{Ct}_{-}} 1$ for positive values (such that the control sample is set to zero) and as the negative inverse for negative values (such that downregulation is presented on a negative scale).

\section{Semi-quantitative polymerase chain reaction (PCR)}

Semi-quantitative PCR was performed to assess mRNA expression for a panel of stem cell and differentiation markers (Mouse/Rat Pluripotent Stem Cell Assessment Primer Pair Panel; R\&D Systems, Abingdon, UK). First strand cDNA synthesis was performed using Superscript III Platinum (Invitrogen) from $100 \mathrm{ng}$ RNA in a $20 \mu \mathrm{L}$ reaction and was followed by RNase treatment. cDNA was diluted in water, and semi-quantitative PCR was performed using Phusion High Fidelity DNA Polymerase (New England BioLabs, Ipswich, MA, USA) according to the manufacturer's instructions, with 35 cycles consisting of annealing at $57^{\circ} \mathrm{C}$, extension at $72{ }^{\circ} \mathrm{C}$, and denaturing at $95{ }^{\circ} \mathrm{C}$. Loading buffer was added to each reaction and $5 \mu \mathrm{L}$ were run on a $1.5 \%(\mathrm{w} / \mathrm{v}$ in $1 \mathrm{X}$ TAE) agarose gel, stained with SYBR Safe (Invitrogen) and visualised with a BioSpectrum 500 (UVP, Cambridge, UK).

\section{Light interferometry}

ESC colony morphology was examined using white light interferometry. Cells were fixed in $2.5 \%(\mathrm{v} / \mathrm{v})$ glutaraldehyde in sodium cacodylate buffer (SCB) for $2 \mathrm{~h}$, washed in SCB and dehydrated in a graded series of ethanol $(50,50,75,80,90$ and $100 \%$; 5 min each) followed by hexamethyldisilazane for $30 \mathrm{~min}$. Specimens were then sputter-coated with gold in preparation for microscopebased white light interferometry (NewView 200, Zygo Corporation, Frankfurt, Germany).

\section{Scanning electron microscopy (SEM) and particle measurement}

In order to observe substrate and cell morphology, scanning electron microscopy was performed. Cells were washed with sodium cacodylate buffer (SCB) and fixed in $2.5 \%$ $(\mathrm{v} / \mathrm{v})$ glutaraldehyde in SCB buffer for $2 \mathrm{~h}$. After fixation, cells were washed in SCB and dehydrated in a graded series of ethanol (50, 50, 75, 80, 90 and $100 \% ; 5$ min each) followed by hexamethyldisilazane for $30 \mathrm{~min}$. Samples were air-dried and sputter-coated with chromium oxide in preparation for SEM (Leo 1525, Carl Zeiss, UK). SEM was performed at $10 \mathrm{kV}$ accelerating voltage. At least 200 particles were measured using Photoshop software (Adobe, Maidenhead, UK).

\section{Cell viability}

Cell viability was determined using a LIVE/DEAD Viability/Cytotoxicity Kit (Invitrogen) according to the manufacturer's instructions. After removing the culture medium, the cells were washed gently in PBS and stained with ethidium homodimer-1 and calcein AM in PBS. After incubating for $20 \mathrm{~min}$, the stain was replaced with fresh medium and the samples were observed under an inverted fluorescence microscope (Olympus, IX51 equipped with a DP70 digital camera, Southend-on-Sea, UK) with FITC (live) and TRITC (dead) filters.

\section{Immunofluorescence}

Immunofluorescence was used to detect proteins of interest in fixed samples. Cells were fixed for $10 \mathrm{~min}$ in $4 \%$ $(\mathrm{w} / \mathrm{v})$ paraformaldehyde, washed in PBS, permeabilised in $0.25 \%(\mathrm{v} / \mathrm{v})$ Triton X-100, washed, and blocked in 5 $\%(\mathrm{v} / \mathrm{v})$ goat serum in PBS-T $(0.1 \%(\mathrm{v} / \mathrm{v})$ Tween-20 in PBS). Cells were incubated in primary antibody diluted in PBS-T overnight at $4{ }^{\circ} \mathrm{C}$ (mouse anti-E-cadherin (Abcam, Cambridge, UK; ab76055, 1/250) and rabbit anti-ZO-1 (Abcam ab59720, 1/50)). Specimens were then washed, incubated in species-specific AlexaFluorconjugated secondary antibodies (Molecular Probes, Eugene, OR, USA) (1/2000) diluted in PBS-T for $1 \mathrm{~h}$ at room temperature in the dark, washed, incubated in $300 \mathrm{nM}$ 4',6-diamidino-2-phenylindole (DAPI), and stored in PBS until confocal microscopy on the same day. Imaging was 

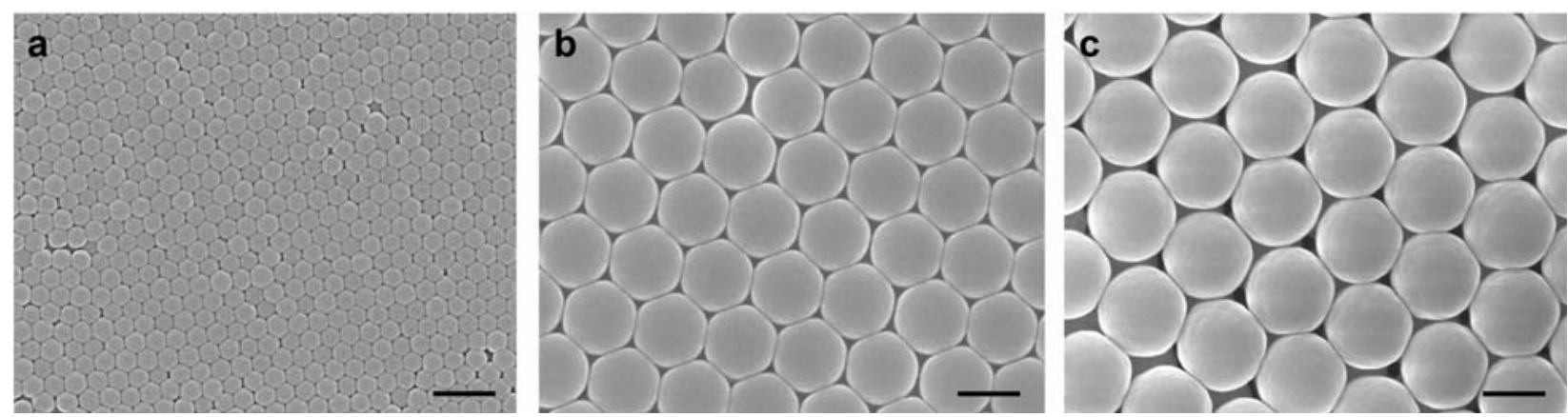

Fig. 1. SEM micrographs of SCC substrates (a) SCC120, (b) SCC400, and (c) SCC600 comprised of particles of $124 \pm 4 \mathrm{~nm}, 430 \pm 4 \mathrm{~nm}$, and $602 \pm 12 \mathrm{~nm}$ diameter, respectively, showing their ordered face-centred cubic packing (scale: $400 \mathrm{~nm}$ ).
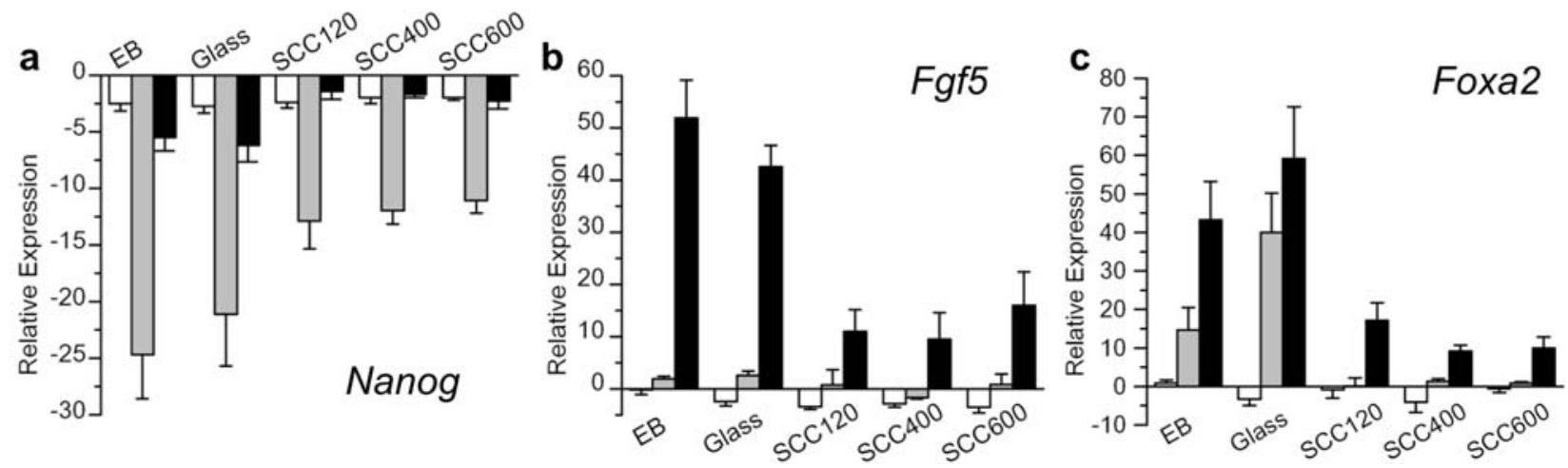

Fig. 2. Quantitative PCR showing the relative gene expression of ESCs grown on SCC substrates compared with ESCs grown on glass slides: (a) Nanog, a stem cell marker was less down-regulated on the SCC substrates than on glass cover slips; (b) Fgf5, a marker of early differentiation, and (c) Foxa2, a marker of endoderm specification were less up-regulated on the SCC substrates than on glass cover slips. White bars: day 5, grey: day 11, black: day $15 . N$ $=3, p<0.05$ considered statistically significant.

performed on an inverted confocal microscope (SP5 MP/ FLIM, Leica, Wetzlar, Germany; UV, Argon (488 nm) and $\mathrm{HeNe}(568 \mathrm{~nm})$ lasers) under standardised gain and offset. Line averages were used. For z-stacks, each stack was $0.84 \mu \mathrm{m}$ thick.

\section{Statistical analysis}

Unless specified otherwise, all data are presented as mean \pm standard deviation. Relative mRNA expression was determined using the $\Delta \Delta \mathrm{C}_{\mathrm{t}}$ method with ESCs at day 0 as the reference sample and Gapdh as the housekeeping gene. Statistical analyses were performed using Student's $t$-test, with $p<0.05$ considered significant, and $p<0.001$ considered highly significant.

\section{Results}

In order to study how the nano- to micrometre scale dimensions of the substrate affect stem cell behaviour, silica colloidal crystal (SCC) substrates were synthesised, characterised by electron microscopy, and coated with collagen to permit cell adhesion. Murine embryonic stem cells (ESCs) were cultured on four types of collagencoated surfaces with varying topography: glass cover slips (as a reference material), $120 \mathrm{~nm}$ diameter SCC substrates, $400 \mathrm{~nm}$ SCC substrates, and $600 \mathrm{~nm} \mathrm{SCC}$ substrates. ESCs were seeded and fed daily with growth medium supplemented with leukaemia inhibitory factor (LIF) and $\beta$-mercaptoethanol for five days, at which point the supplements were removed and cells were fed daily in differentiation medium for up to another ten days. The effect of topography on the expression of stem cell and early differentiation genes was investigated using polymerase chain reaction, and stem cell colony features were probed using white light interferometry and scanning electron microscopy (SEM). Finally, protein expression related to epithelialisation was examined using immunofluorescence.

\section{Substrate fabrication and collagen adsorption}

The size of the particles and the substrate topography were evaluated using scanning electron microscopy (SEM) (Fig. 1). The SCC substrates were comprised of monodisperse (polydispersity index $(\mathrm{PDI})=1.00)$ microspheres with ordered face-centred cubic packing up to an area of $1 \mathrm{~cm}^{2}$ (Fig. 1a-c). The mean sizes and standard deviations of the particles were $124 \pm 4 \mathrm{~nm}, 430 \pm 4 \mathrm{~nm}$, and $602 \pm 12 \mathrm{~nm}$ for SCC120, SCC400, and SCC600, respectively. The specific surface areas of SCC120, SCC400, and SCC600 were $68.1,38.7$ and $26.2 \mathrm{~m}^{2} / \mathrm{g}$, as measured by nitrogen absorption. A collagen quantification assay confirmed 
that Type I collagen had adsorbed to the SCC substrates and glass cover slips in approximately equal amounts (maximum two-fold deviation between samples; $N=6$ ).

\section{Gene expression}

Quantitative polymerase chain reaction (qPCR)

The gene expression of selected stem cell and early differentiation markers was determined using qPCR (Fig. 2). We found significant changes in gene expression in cells grown on the SCC substrates compared to glass cover slips. The transcript levels of ESCs at day zero prior to seeding onto the experimental substrates were set to zero, and all data are shown relative to that value with Gapdh as an internal reference transcript. Any negative values (corresponding to down-regulation) are displayed as the negative inverse. Nanog, a transcription factor involved in the self-renewal of undifferentiated ESCs, was significantly down-regulated on all substrates after eleven days in culture (six days following the removal of LIF and $\beta$-mercaptoethanol from the culture medium) (Fig. 2a). The mRNA levels in cells seeded on the three SCC substrates (SCC120, SCC400, and SCC600) were less down-regulated than on glass and in embryoid bodies. By day fifteen, the levels of Nanog mRNA remained significantly down-regulated in cells on all substrates but had returned to levels closer to the day zero levels. Fgf5, which is expressed in the primitive ectoderm but not in undifferentiated ESCs, is a marker of early differentiation. Its expression increased with culture time and was significantly greater in cells within EBs and on the glass substrates than in cells cultured on SCCs (Fig. 2b). Foxa2, a mesendodermal marker expressed during gastrulation followed a similar pattern of expression to Fgf5. It was upregulated in cells on the SCC substrates but to a much lesser extent than cells on the glass cover slips (Fig. 2c). Together, these data suggested that cells on the SCC substrates were promoting an undifferentiated phenotype more than glass cover slips, which led us to further investigate lineage specification and colony morphology to better understand how topography may be influencing stem cell behaviour. Because the three SCC substrates had a similar effect on the ESCs, we continued our gene expression experiments with only the SCC400 substrate.

Semi-quantitative polymerase chain reaction

With the quantitative PCR demonstrating significant changes to some stemness and early differentiation

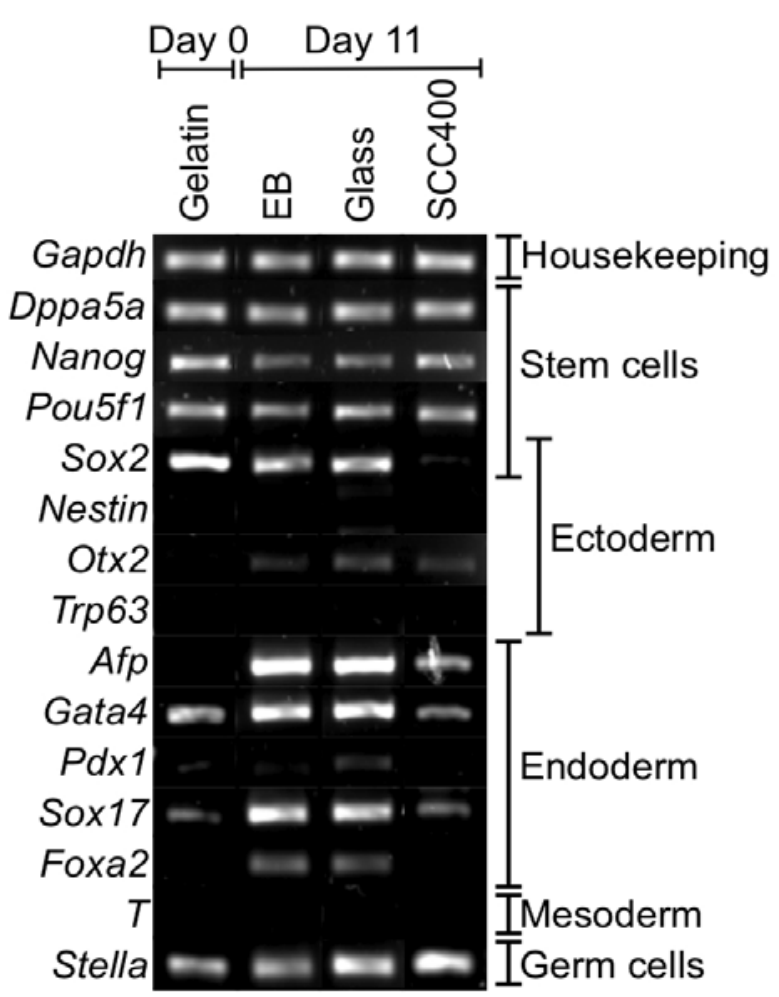

Fig. 3. Semi-quantitative PCR of stem cell and differentiation markers in day 0 ESCs (on gelatin), and day $11 \mathrm{EBs}$, ESCs on glass slides, and ESCs on SCC400 substrates. Transcription levels of the stem cell markers Dppa5a, Nanog, and Pou5f1 were preserved in all samples. The stem cell and ectoderm marker, Sox2, was down-regulated on the SCC400 substrate. Endoderm markers Afp, Gata4, Sox17 and Foxa2, were all closer to undifferentiated ESC levels in cells cultured on the SCC400 substrates than in cells in EBs or on glass cover slips.

markers, we used a panel of primers to take a closer look at the fate of the differentiating cells. Fig. 3 shows the amplicons for an array of genes, including Gapdh (a housekeeping gene), Dppa5a, Nanog, Pou5f1, and Sox2 (ESC markers), Sox2, Nestin, Otx2, and Trp63 (ectoderm), Afp, Gata4, Pdx1, Sox17, and Foxa2 (endoderm), T (mesoderm), and Stella (germ cells). ESCs at day 0 (the first column) had a high expression of all stem cell markers, which was maintained in EBs and ESCs cultured
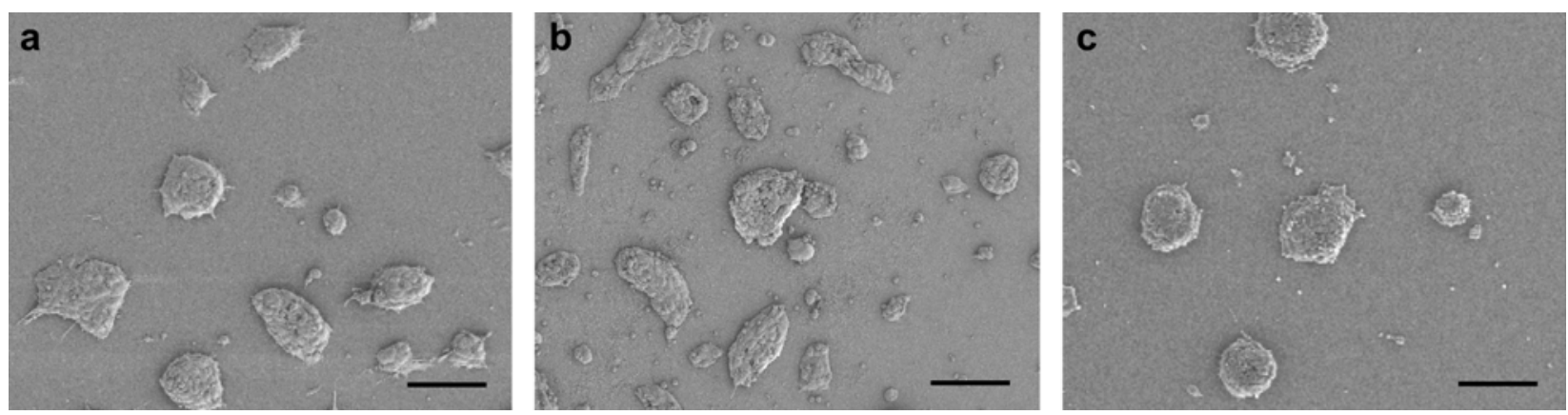

Fig. 4. SEM micrograph of ESC colonies five days after seeding on (a) SCC120, (b) SCC400, and (c) SCC600 showed similar colony morphology, which led us to continue experiments with only the SCC400 substrate (scale: $100 \mu \mathrm{m}$ ). 
a

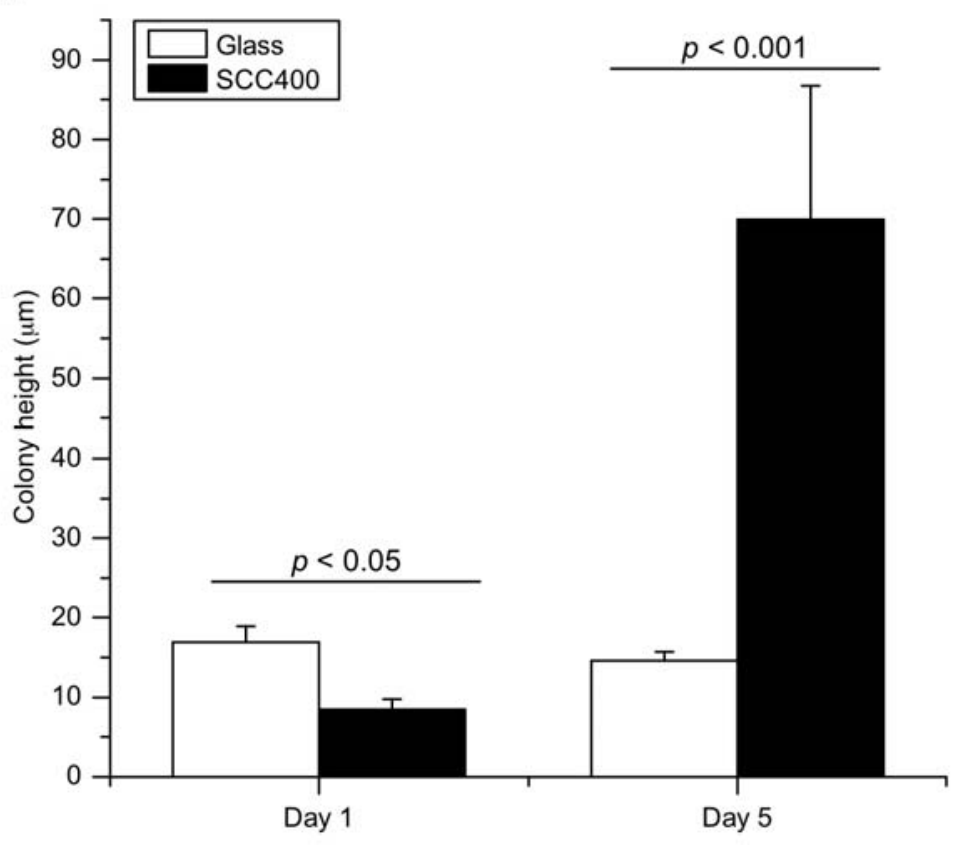

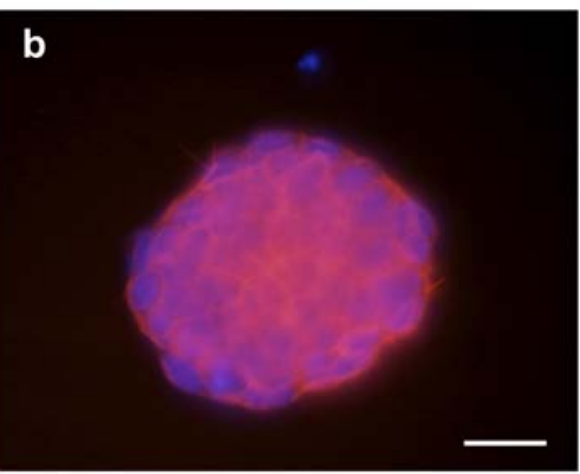

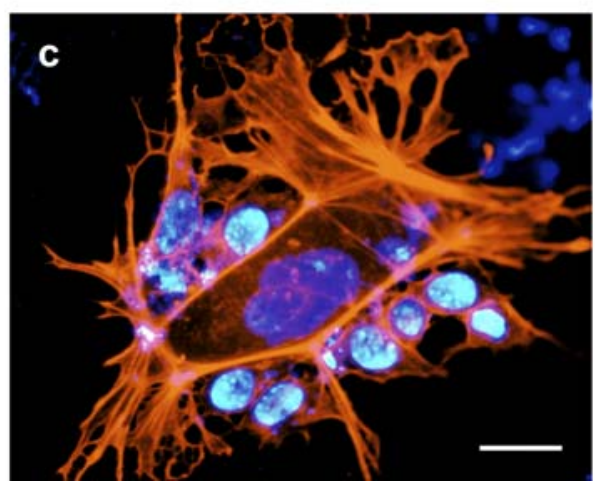

Fig. 5. (a) White light interferometry was used to measure the colony heights on day 1 and day 5 after cell seeding. By day 5, the colonies on SCC400 were comprised of multiple layers of cells, but on glass cover slips, the cells were growing in a monolayer $(N=25, p<0.05$ considered statistically significant). Cytoskeleton staining in an ESC colony on (b) SCC400 demonstrated strikingly different cytoskeletal morphology compared to on (c) a glass cover slip, which exhibited highly elongated stress fibres (red: phalloidin, blue: DAPI, scale: $50 \mu \mathrm{m}$ ).

on both glass cover slips and SCC400 substrates after 11 days in culture. Only Sox2 expression was diminished on the SCC400 substrates, though its expression is also indicative of ectoderm specification. Most interestingly, the expression of the endoderm markers Afp, Gata4, Sox17, and Foxa2 all increased in EBs and ESCs on glass, but were found only at low levels in ESCs on the SCC400, suggesting these topographies are particularly active in repressing endoderm specification.

\section{ESC colony morphology}

Scanning electron microscopy

While an adsorbed layer of Type 1 collagen was required for cells to attach to the SCC substrates but not to the glass cover slips, both types of substrates were coated with collagen prior to cell seeding. With this collagen pre-incubation, ESCs initially attached on all substrates and proliferated to form colonies. Under phase contrast microscopy, only cells on the SCC120 and SCC400 surfaces were visible, as SCC600 did not transmit light. Therefore, white light interferometry and scanning electron microscopy were used to closely examine colony morphology. Cells on the SCC120, SCC400 and SCC600 substrates five days after seeding formed spherical colonies with similar morphology on all substrates (Fig. 4a-c) and exhibited similar central pit formation at a later time point.

\section{White light interferometry}

To further characterise the effects of topography on stem cell colony morphology, we used white light interferometry and found varying colony height between the SCC substrates and the glass cover slips (Fig. 5a). One day after seeding, the colonies had a mean height of $8.4 \pm 1.4 \mu \mathrm{m}$ on the SCC400 substrates, and $16.9 \pm 2.1 \mu \mathrm{m}$ on glass cover slips. While this difference was statistically significant ( $p$ $<0.05$ ), it only represented a height difference of one or two cells. On both substrates, the colony height on day one was representative of approximately 2-3 cells, given an estimated post-fixation cell diameter of 5-10 $\mu \mathrm{m}$, as measured from SEM micrographs.

After five days in culture, there was a highly statistically significant $(p<0.001)$ difference between the height of colonies on the SCC400 substrates and the glass cover slips, representing a height difference of many cells. The colony height on the SCC substrates had increased to 69.9 $\pm 16.9 \mu \mathrm{m}$, while it had decreased on the glass cover slips to $14.6 \pm 1.1 \mu \mathrm{m}$. These data suggest that cell spreading was reduced by the structure of the SCC surface, which subsequently led us to investigate stress fibre morphology.

\section{Phalloidin histochemistry}

The distribution of phalloidin staining further demonstrated the strikingly different colony morphology on SCC 

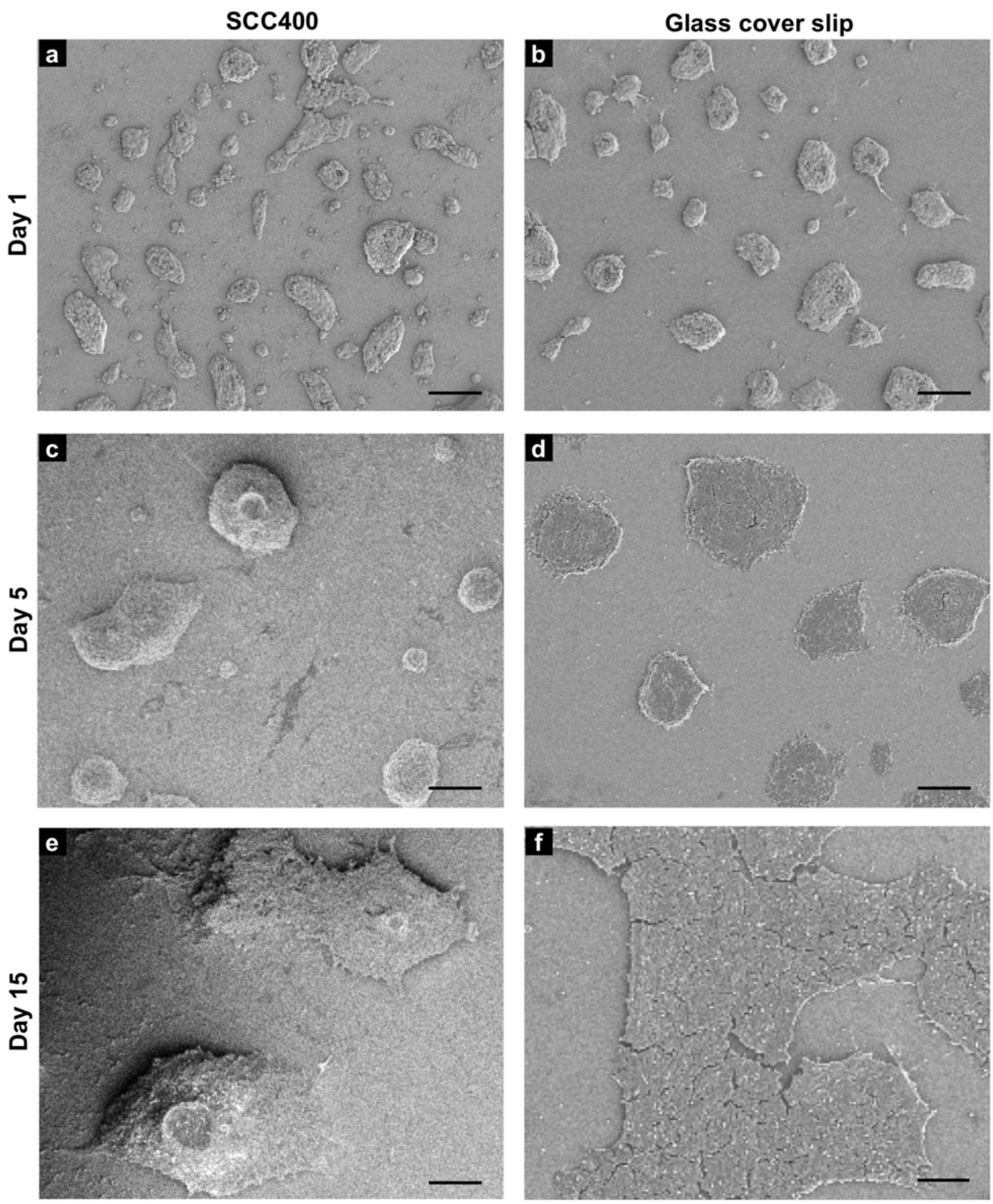

Fig. 6. SEM micrographs of ESC colonies at day 1 on (a) an SCC400 substrate and (b) a glass cover slip, day 5 on (c) an SCC400 substrate and (d) a glass cover slip, and day 15 on (e) an SCC400 substrate and (f) a glass cover slip. Colonies on the SCC400 substrates formed rounder, more spherical colonies than on glass cover slips and began to form central pits by day 5 (scale: $100 \mu \mathrm{m}$ ).

substrates compared to the glass cover slips. Phalloidin stains the actin filaments and is therefore useful for visualising cell morphology. Five days after seeding, the colonies on SCC substrates retained their spherical morphology, resembling undifferentiated ESCs (Fig. 5b).
The colonies were comprised of many round cells with diffuse cytoskeletons and an absence of stress fibres. In stark contrast, the cells grown on glass cover slips had spread and were in a monolayer with highly established and elongated stress fibres (Fig. 5c). 


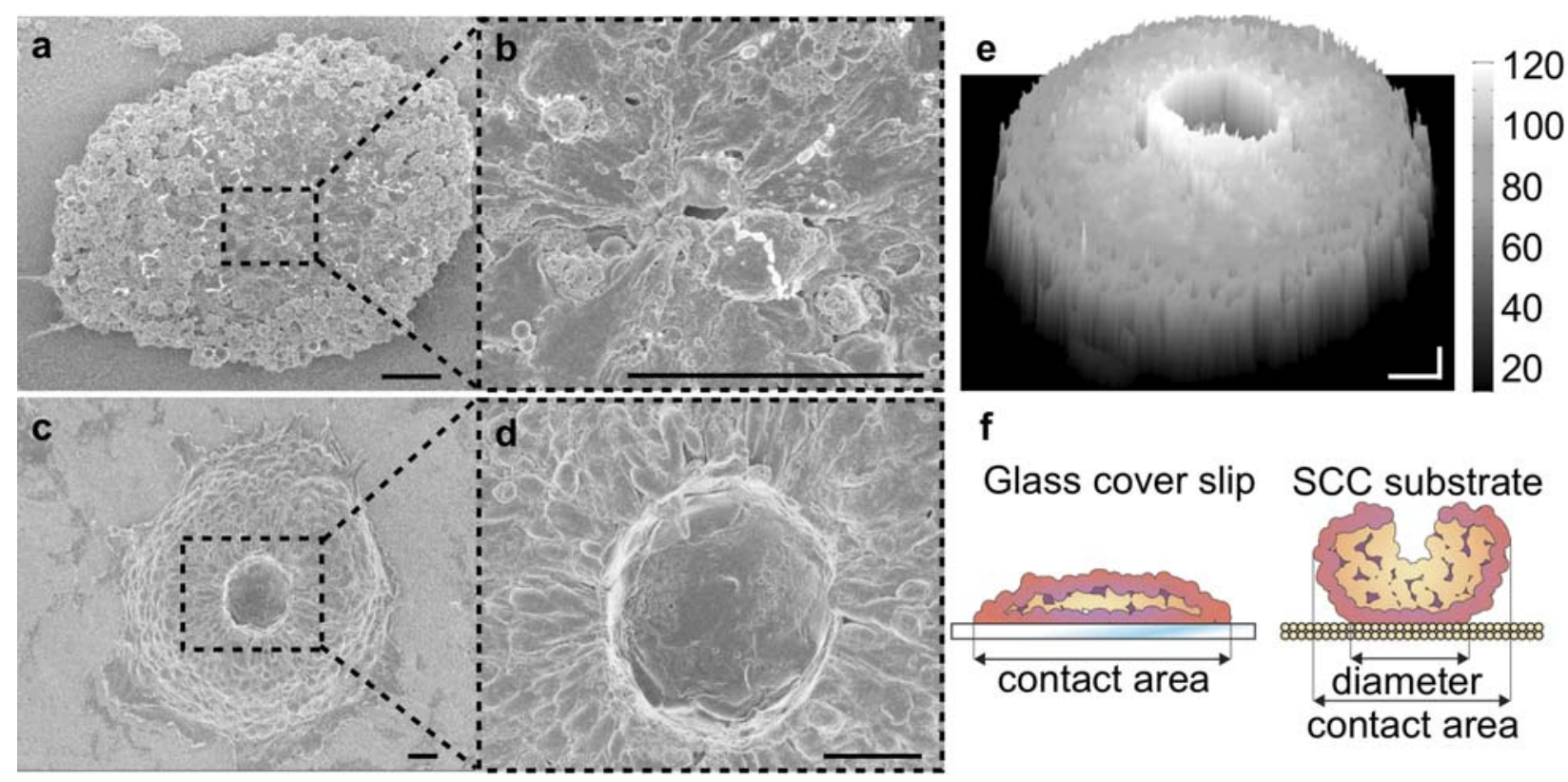

Fig. 7. ESCs grown on the SCC substrates develop central pits. The pits begin to form around (a) day 5 (high magnification of boxed area shown in (b)) and grow larger until (c) day 15 (high magnification of boxed area shown in $(\mathbf{d}))$ (scale: $20 \mu \mathrm{m})$. The presence of the pit was confirmed using microscope-based white light interferometry, where (e) shows a pit at day 15 on the SCC400 substrate (scale: $50 \mu \mathrm{m}$, z-axis in $\mu \mathrm{m}$ ). The schematic in (f) depicts ESC colony morphology on a glass cover slip and SCC substrate.

\section{Central pit formation}

In addition to the differences seen in cell spreading and colony height, SEM micrographs and white light interferometry of ESC colonies growing on SCC substrates revealed another interesting phenomenon not observed by phase contrast microscopy. Approximately five days after seeding, ESC colonies on the SCC substrates began to develop increasingly large cavities located in the centre of the colonies, which we have termed "central pits". These pits increased in size and prevalence up to fifteen days after seeding, when virtually all colonies contained a large pit. This phenomenon was never observed on glass cover slips.

\section{Scanning electron microscopy}

Fig. 6 illustrates the changing appearance of ESC colonies on an SCC400 substrate compared to a glass cover slip. One day after seeding, ESCs formed colonies with similar spherical morphology on SCC400 substrates as on glass cover slips (Fig. 6a-b). Five days after culture in growth medium, the emergence of a single central pit was observed in some ESC colonies on the SCC substrates (Fig. 6c) but never on the glass cover slips (Fig. 6d). Between days five and fifteen on the SCC substrates, more colonies developed a central pit, and the size of the pits increased (Fig. 6e); in contrast, cells on the glass cover slip grew in a monolayer and did not form central pits (Fig. 6f).

The formation of a central pit on an SCC400 substrate is shown in Fig. 7. Between days one and five after seeding, the cells at the centre of the colonies aligned radially around the prospective central pit (Fig. 7a-b). Between days five and fifteen, a small ingress grew in size and depth, leading to the formation of a central pit (Fig. 7c-d). In colonies containing a central pit, the cells around the pit were elongated and columnar in shape, whereas those on the colony edge remained spherical (Fig. 7c-d).

\section{White light interferometry}

The central pits were not simply artefacts of SEM imaging, as evidenced by their absence in colonies grown on glass cover slips. In addition, images obtained by microscopebased white light interferometry revealed the surface profile of a colony with similar morphology to those in the SEM micrographs (Fig. 7e). ESC colonies on the SCC substrates with central pits were typically 100-300 $\mu \mathrm{m}$ in diameter, with a minimum height of approximately $80 \mu \mathrm{m}$. Fig. $6 \mathrm{f}$ shows a typical central pit with a depth of $33 \mu \mathrm{m}$, which is equivalent to the diameter of 3-7 single ESCs.

\section{Cell viability}

Cell viability in the colonies was investigated to determine whether cell death in the centre of the large colonies and the subsequent formation of a necrotic core was a possible explanation for the central pits. However, the results from a cell viability assay did not support this hypothesis (Fig. 8 ), though the location and time-course of cell death was different in colonies on the SCC substrates and glass cover slips. On the glass cover slips, many dead cells were observed in the centre of the colonies one day after seeding (Fig. 8b), but none were seen in the centre of the colonies on any of the SCC substrates (Fig. 8a).

\section{Immunofluorescence}

Finally, we examined whether cells around the upper layers of the colonies were epithelial cells using immunofluorescence for epithelial markers. This hypothesis was based on the examination of scanning 

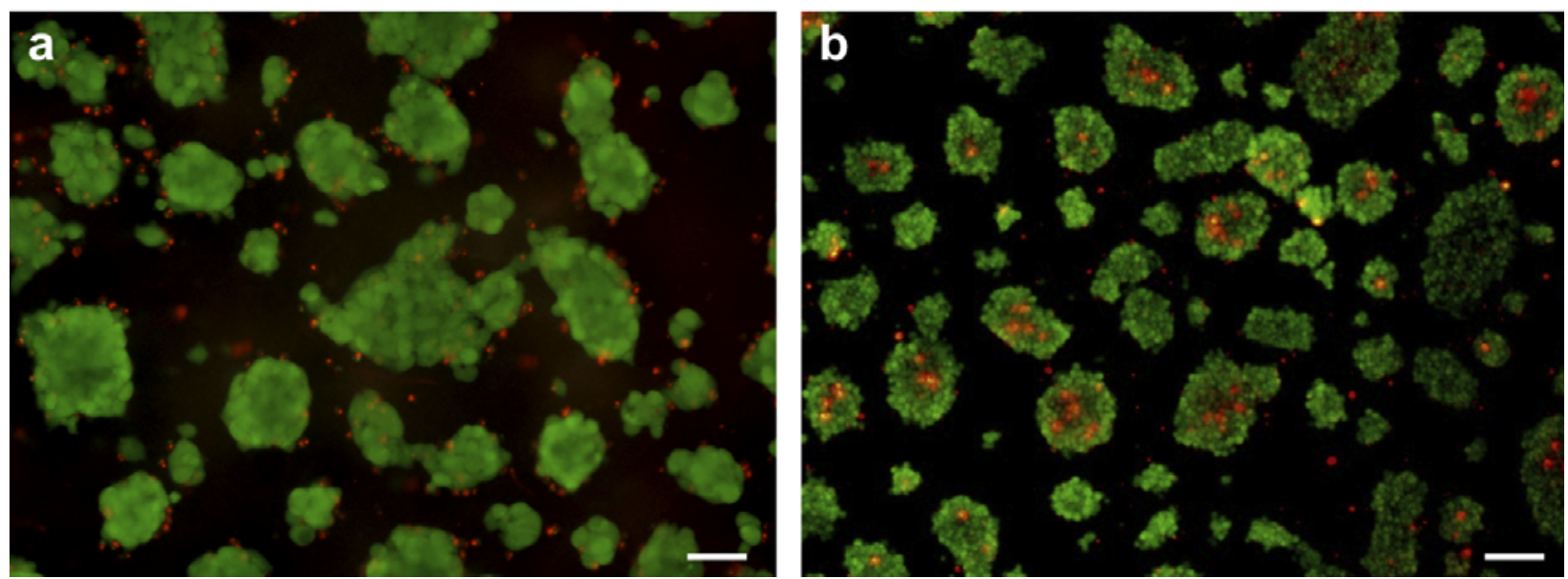

Fig 8. LIVE/DEAD staining (green: live cells, red: dead cells) indicates the central pits in the colonies on SCC substrates were not caused by the death of cells. ESC colonies on (a) SCC400 and (b) a glass cover slip on day 3. There were no dead cells present in the centres of the colonies on the SCC400 substrates but they were present on glass cover slips (scale: $100 \mu \mathrm{m}$ ).

electron micrographs of colonies with central pits where the cells around the pit had organised, epithelial-like morphology. E-cadherin is found in cell-to-cell junctions of epithelial tissues, and its presence increases the strength of cellular adhesions within a tissue. The distribution of epithelial cells was revealed by immunofluorescence staining of E-cadherin and ZO-1, a protein associated with intercellular tight junctions. Fig. 9 shows the strongest expression for both E-cadherin and ZO-1 was at the periphery of the colony base on the SCC400 substrate. From the base to the top of the colony, E-cadherin and ZO-1 expression decreased gradually but the strongest expression in every cross-section remained at the periphery (Fig. 8b-e), suggesting that cells in the outside layer of the colony are expressing epithelial markers. DAPI staining of cell nuclei showed the area at the base of the colony was much smaller than that of other layers, confirming that the colonies were not spreading on the SCC400 substrates to the same extent as they did on glass cover slips.

\section{Discussion}

In this study, we synthesised silica colloidal crystal (SCC) microspheres with diameters of approximately 120, 400 and $600 \mathrm{~nm}$ and fabricated substrates from them with topographical features of the same sizes (Fig. 1). The total amount of pre-adsorbed collagen was similar on each substrate and the cells therefore had a similar quantity of collagen available for binding. In order to determine how the substrates influence murine embryonic stem cell behaviour, we examined gene expression, colony morphology and protein expression on the SCC substrates and on glass cover slips.

We first observed that cells cultured on SCC substrates maintain gene expression more similar to ESCs than cells on glass cover slips or in embryoid bodies (Fig. 2). We quantified that Nanog was down-regulated, and Fgf5 and Foxa2 up-regulated, to a lesser extent in cells on SCCs than either on glass cover slips or in EBs, indicating that population-wide differentiation is less pronounced in SCC substrate specimens. Looking at markers for specification into ectoderm, endoderm, or mesoderm, we found that the SCC400 topography appeared to specifically inhibit the endoderm differentiation that occurred in EBs and in ESCs cultured on glass cover slips after eleven days in culture. The substrate effects on these differentiation markers may have been through a number of mechanisms. Firstly, we found that SCC substrates inhibited ESC colony spreading. Since cell spreading, stimulated by ECM components promotes ESC differentiation by the activation of integrin signalling pathways (Hayashi et al., 2007) and conversely, the reduction of cell-substrate contacts promotes selfrenewal (Chowdhury et al., 2010), we suggest that culture on the SCC substrates, which inhibit spreading, in turn repress the effectors of integrin signalling, and therefore limits differentiation. A thorough review of the effects of feature spacing on integrin adhesion by Biggs et al. also suggests that features in the size range we studied may disrupt integrin binding, which is consistent with the diminished colony spreading we observed (Biggs et al., 2010). Andersson et al. found that human bladder cells on hemispherical structures appeared less spread than those on flat samples with a corresponding decrease in cytokine production (Andersson et al., 2003). Additionally, the increased paracrine growth-factor signalling between ESCs in more three-dimensional, tightly packed colonies on the SCC substrates may also stimulate self-renewal. Dani et al. showed that even in the absence of LIF, paracrine factors in ESC colonies can stimulate self-renewal (Dani et al., 1998). Of course, this is also true in EBs, but since EBs are in the region of $300-1000 \mu \mathrm{m}$ in thickness (compared to approximately $80 \mu \mathrm{m}$ for colonies on the SCC substrates), other factors such as increased cell migration, nutrient depletion, and distance-dependent paracrine signalling antagonistic to self-renewal may obscure this effect.

The most differential feature noted was the reproducible formation of a central pit in the centre of ESC colonies 


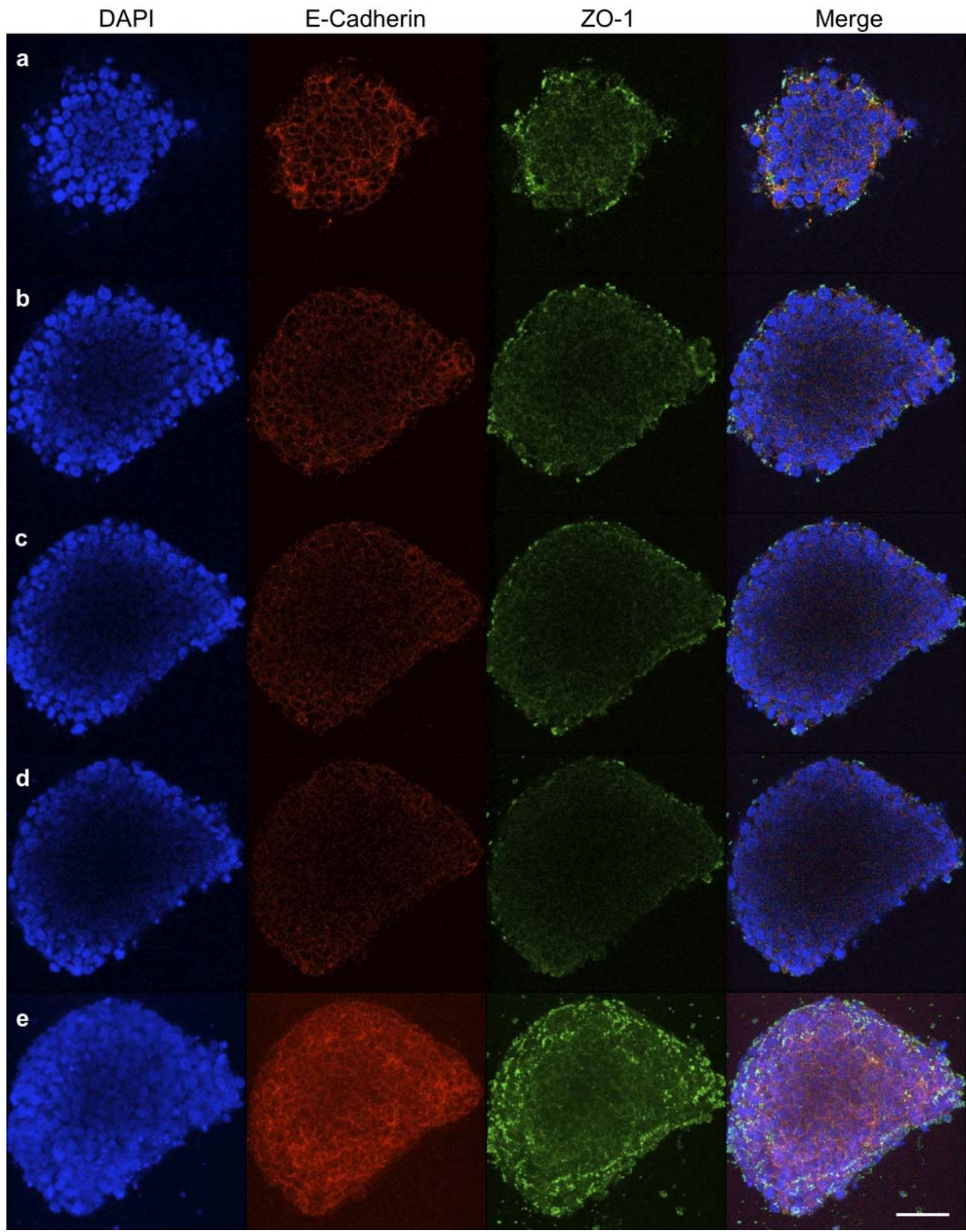

Fig. 9. Immunofluorescence demonstrating a central pit. The colony in contact with (a) the SCC400 substrate has a small area that increases (b) $15 \mu \mathrm{m}$, (c) $30 \mu \mathrm{m}$ and (d) $35 \mu \mathrm{m}$ from the substrate. DAPI staining indicates a lack of nuclei in the centre of the colony. E-cadherin staining is strongest at the bottom of the colony (a) and becomes more diffuse towards the middle and top (b, c, d). ZO-1 staining is strongest at the bottom of the colony (a) and the periphery (b, c, e). A merge of all z-stacks $(0.84 \mu \mathrm{m}$ each) is shown in (e) (scale: $50 \mu \mathrm{m})$.

cultured on SCC substrates (Figs. 6 and 7). This characteristic was observed by both scanning electron microscopy and white light interferometry. A similar phenomenon in vitro has been observed by Behr et al., when rhesus monkey ESCs were cultured on mouse embryonic fibroblast (MEF) feeder layers (Behr et al., 2005). Immunofluorescence indicated their cells were undergoing an epithelial-mesenchymal transition and the 
central pit formation was attributed to the presence of the feeder layer. In our study, we showed that central pit formation was induced by the SCC substrates. We used white light interferometry and viability assay staining to further characterise the colonies with central pits. Colonies with a central pit were always above $80 \mu \mathrm{m}$ in height, which suggests the importance of the number of cell-cell contacts in dictating the formation of pits. We initially considered that pit formation may have been the result of cell death at the centre of the colonies cultured on SCC substrates; however, no cell death in the pit location was observed. Without any evidence supporting cell death as a cause for central pit formation, we instead suggest that the lack of spreading and subsequently enhanced cell-cell contacts in the spherical colonies promoted the central pit formation.

Cells in the top layer of the colony around the central pit were columnar in shape and radially arranged. Epithelial markers E-cadherin and ZO-1 were expressed more strongly around the edge of the colony than in the centre (Fig. 9). Columnar cells showing epithelial-like characteristics were also observed by Ullmann et al. in the top layer of human embryonic stem cell colonies when the epithelial-mesenchymal transition (EMT) process was taking place, although no central pit morphology was reported (Ullmann et al., 2007). In our study, it is possible that a process similar to EMT is taking place around the central pit, where cells lose expression of epithelial markers and begin to assume a mesenchymal, migratory phenotype. Immunofluorescence for both epithelial and mesenchymal markers at various time points would have to be performed on these substrates to assess whether an EMT is occurring.

Modern technologies have made it possible to fabricate diverse, complex, and reproducible topographies across large surface areas. In combination with other substrate cues known to affect cell behaviour, topographical features could potentially guide stem cell differentiation strongly enough to create the homogenous cell population needed in regenerative medicine applications. Future work on the effects of these substrates on ESC self-renewal would complement the morphology observations in this study and reconcile the apparent decrease in differentiation markers with the appearance of a morphological feature reminiscent of an early differentiation event.

\section{Conclusions}

In conclusion, we fabricated substrates of well-defined and highly reproducible nano- to micrometre topography and studied their effects on ESCs. We found that substrate topography can influence both the shape and fate of differentiating colonies of ESCs. Specifically, cells grown on the substrates with topography retained a spherical colony morphology and eventually developed a central pit. In this work, we have shown the profound effects substrate topography can have on ESCs and this system provides a possible method of visualising and characterising cell arrangements in early mammalian development and for better understanding the effects of topography on embryonic stem cells.

\section{Acknowledgements}

Microscopy was performed in the Facility for Imaging by Light Microscopy (FILM) at Imperial College London. We thank Dr. Rekha Nair for critical review of the manuscript and Dr. Elsie Place for assistance with the figures. LJ thanks the Royal Society for an Incoming Postdoctoral Fellowship to work with MMS. NDE thanks the Medical Research Council, UK, for a postdoctoral career development fellowship. MMS thanks the ERC starting investigator grant "Naturale" for funding.

\section{References}

Andersson A-S, Bäckhed F, Euler von A, RichterDahlfors A, Sutherland D, Kasemo B (2003) Nanoscale features influence epithelial cell morphology and cytokine production. Biomaterials 24: 3427-3436.

Behr R, Heneweer C, Viebahn C, Denker H-W, Thie M (2005) Epithelial-mesenchymal transition in colonies of rhesus monkey embryonic stem cells: a model for processes involved in gastrulation. Stem Cells 23: 805-816.

Benoit DSW, Schwartz MP, Durney AR, Anseth KS (2008) Small functional groups for controlled differentiation of hydrogel-encapsulated human mesenchymal stem cells. Nat Mater 7: 816-823.

Biggs MJP, Richards RG, Dalby MJ (2010) Nanotopographical modification: a regulator of cellular function through focal adhesions. Nanomedicine 6: 619633.

Chai C, Leong KW (2007) Biomaterials approach to expand and direct differentiation of stem cells. Mol Ther 15: 467-480.

Chen CS, Mrksich M, Huang S, Whitesides GM, Ingber DE (1997) Geometric control of cell life and death. Science 276: $1425-1428$.

Chowdhury F, Li Y, Poh Y-C, Yokohama-Tamaki T, Wang N, Tanaka TS (2010) Soft substrates promote homogeneous self-renewal of embryonic stem cells via downregulating cell-matrix tractions. PLoS ONE 5: e15655.

Curran JM, Chen R, Hunt JA (2005) Controlling the phenotype and function of mesenchymal stem cells in vitro by adhesion to silane-modified clean glass surfaces. Biomaterials 26: 7057-7067.

Curran JM, Stokes R, Irvine E, Graham D, Amro NA, Sanedrin RG, Jamil H, Hunt JA (2010) Introducing dip pen nanolithography as a tool for controlling stem cell behaviour: unlocking the potential of the next generation of smart materials in regenerative medicine. Lab Chip 10: 1662-1670.

Curran JM, Pu F, Chen R, Hunt JA (2011) The use of dynamic surface chemistries to control MSC isolation and function. Biomaterials 32: 4753-4760.

Dalby MJ, Gadegaard N, Tare R, Andar A, Riehle MO, Herzyk P, Wilkinson CDW, Oreffo ROC (2007) The control of human mesenchymal cell differentiation using nanoscale symmetry and disorder. Nat Mater 6: 997-1003. 
Dani C, Chambers I, Johnstone S, Robertson M, Ebrahimi B, Saito M, Taga T, Li M, Burdon T, Nichols J, Smith A (1998) Paracrine induction of stem cell renewal by LIF-deficient cells: a new ES cell regulatory pathway. Dev Biol 203: 149-162.

Discher DE, Mooney DJ, Zandstra PW (2009) Growth factors, matrices, and forces combine and control stem cells. Science 324: 1673-1677.

Engler AJ, Sen S, Sweeney HL, Discher DE (2006) Matrix elasticity directs stem cell lineage specification. Cell 126: 677-689.

Evans MJ, Kaufman MH (1981) Establishment in culture of pluripotential cells from mouse embryos. Nature 292: 154-156.

Evans ND, Minelli C, Gentleman E, LaPointe V, Patankar SN, Kallivretaki M, Chen X, Roberts CJ, Stevens MM (2009) Substrate stiffness affects early differentiation events in embryonic stem cells. Eur Cell Mater 18: 1-13.

Frantz C, Stewart KM, Weaver VM (2010) The extracellular matrix at a glance. J Cell Sci 123: 4195-4200.

Gaudet C, Marganski WA, Kim S, Brown CT, Gunderia V, Dembo M, Wong JY (2003) Influence of type I collagen surface density on fibroblast spreading, motility, and contractility. Biophys J 85: 3329-3335.

Gilbert PM, Havenstrite KL, Magnusson KEG, Sacco A, Leonardi NA, Kraft P, Nguyen NK, Thrun S, Lutolf MP, Blau HM (2010) Substrate elasticity regulates skeletal muscle stem cell self-renewal in culture. Science 329: 1078-1081.

Hayashi Y, Furue MK, Okamoto T, Ohnuma K, Myoishi Y, Fukuhara Y, Abe T, Sato JD, Hata R-I, Asashima M (2007) Integrins regulate mouse embryonic stem cell selfrenewal. Stem Cells 25: 3005-3015.

Keselowsky BG, Collard DM, García AJ (2005) Integrin binding specificity regulates biomaterial surface chemistry effects on cell differentiation. Proc Natl Acad Sci USA 102: 5953-5957.

Kilian KA, Bugarija B, Lahn BT, Mrksich M (2010) Geometric cues for directing the differentiation of mesenchymal stem cells. P Natl Acad Sci USA 107: $4872-$ 4877.

Lee ST, Yun JI, Jo YS, Mochizuki M, van der Vlies AJ, Kontos S, Ihm JE, Lim JM, Hubbell JA (2010) Engineering integrin signaling for promoting embryonic stem cell selfrenewal in a precisely defined niche. Biomaterials 31 : 1219-1226.
McBeath R, Pirone DM, Nelson CM, Bhadriraju K, Chen CS (2004) Cell shape, cytoskeletal tension, and RhoA regulate stem cell lineage commitment. Dev Cell 6: 483-495.

McMurray RJ, Gadegaard N, Tsimbouri PM, Burgess KV, McNamara LE, Tare R, Murawski K, Kingham E, Oreffo ROC, Dalby MJ (2011) Nanoscale surfaces for the long-term maintenance of mesenchymal stem cell phenotype and multipotency. Nat Mater 10: 637-644.

Rozario T, DeSimone DW (2010) The extracellular matrix in development and morphogenesis: a dynamic view. Dev Biol 341: 126-140.

Salasznyk RM, Williams WA, Boskey A, Batorsky A, Plopper GE (2004) Adhesion to vitronectin and collagen I promotes osteogenic differentiation of human mesenchymal stem cells. J Biomed Biotechnol 2004: 2434.

Stevens MM, George JH (2005) Exploring and engineering the cell surface interface. Science 310: 11351138 .

Stöber W, Fink A, Bohn E (1968) Controlled growth of monodisperse silica spheres in the micron size range. $\mathrm{J}$ Colloid Interface Sci 26: 62-69.

Ullmann U, In't Veld P, Gilles C, Sermon K, de Rycke M, van de Velde H, van Steirteghem A, Liebaers I (2007) Epithelial-mesenchymal transition process in human embryonic stem cells cultured in feeder-free conditions. Mol Hum Reprod 13: 21-32.

Unadkat HV, Hulsman M, Cornelissen K, Papenburg BJ, Truckenmüller RK, Post GF, Uetz M, Reinders MJT, Stamatialis D, van Blitterswijk CA, de Boer J (2011) An algorithm-based topographical biomaterials library to instruct cell fate. Proc Natl Acad Sci USA 108: 1656516570 .

Weber LM, Hayda KN, Haskins K, Anseth KS (2007) The effects of cell-matrix interactions on encapsulated beta-cell function within hydrogels functionalized with matrix-derived adhesive peptides. Biomaterials 28: $3004-$ 3011.

Editor's Note: All questions/comments from the reviewers were answered by the authors as text changes. Hence, there is no Discussion with Reviewers. 